\title{
ON INCOMPLETE AND BALANCED INCOMPLETE BLOCK DESIGNS
}

KULENDRA N. MAJINDAR

By an incomplete block design we will mean an arrangement of $v$ different varieties of objects in $b$ distinct blocks or sets such that a block does not contain all the varieties and a variety appears at most once in a block. An incomplete block design which satisfies two extra conditions, viz. (i) every pair of varieties occurs together in $\lambda \neq 0$ of the blocks and (ii) each block contains the same number of objects, say $k$, is called a balanced incomplete block (b.i.b. for conciseness) design. A b.i.b. design with $b=v$ is sometimes called a $v-k-\lambda$ configuration.

It is known [1] and can be seen below that in a b.i.b. design every variety occurs the same number, say $r$, of times. Clearly then $b k=v r$, $\lambda(v-1)=r(k-1)$. For a b.i.b. design Fisher's inequality $b \geqq v$ must also hold [1].

An incomplete block design satisfying condition (i) is not necessarily a b.i.b. design. Ryser [3] has proved (in an essentially equivalent form) that if in a symmetrical incomplete block design (that is to say, one in which $b=v$ ) every variety appears a constant number of times, say $r$, also condition (i) holds, then $\lambda(v-1)=r(k-1)$ and (ii) holds and it is a symmetrical b.i.b. design. We give here conditions under which any incomplete block design becomes a b.i.b. design. An extension of Ryser's result in another direction has been given in [2].

THEOREM 1. If in an incomplete block design with $v$ varieties and $b$ blocks, every pair of varieties occurs together in $\lambda \neq 0$ of the blocks, moreover

$$
t(t-b) \geqq \lambda(v-1) v b
$$

where $t$ denotes the total number of objects in the whole design, then it is a b.i.b. design.

Proof. Let the $i$ th block contain $x_{i}$ objects, $i=1,2, \cdots, b$. Then $\sum x_{i}=t$. Considering pairs of varieties we get $\sum x_{i}\left(x_{i}-1\right)=\lambda(v-1) v$. Thus $\sum x_{i}^{2}=t+\lambda(v-1) v$. By Cauchy's inequality we have $t^{2} \leqq(t+\lambda(v-1) v) b$. Now (1) makes $t^{2}=(t+\lambda(v-1) v) b$. Thus the inequality reduces to an equality and this can happen if and only if all the $x_{i}$ 's are equal, to $k$ say. Hence each of the blocks contains $k$

Received by the editors November 29, 1961. 
objects. So the incomplete block design is a b.i.b. design.

If the $i$ th variety occurs $r_{i}$ times in a b.i.b. design, then, as it has to appear together with each of the other varieties in $\lambda$ of the blocks, we have $k r_{i}=r_{i}+\lambda(v-1)$ so that $r_{i}=\lambda(v-1) /(k-1)$. Therefore all the varieties appear with the same frequency.

In general, an incomplete block design in which all the varieties occur with the same frequency and which satisfies condition (i) of the first paragraph fails to be a b.i.b. design. Further conditions are necessary to make it a b.i.b. design. The following theorem gives one set of such conditions.

THEOREM 2. If in an incomplete block design with $v$ varieties and $b$ blocks, every pair of varieties occurs together in $\lambda \neq 0$ of the blocks and each variety appears $r$ times in the design, furthermore for some positive integer $v^{\prime}$ with $v^{\prime} \leqq v$

$$
v^{\prime} r^{2} \geqq\left(r+\lambda\left(v^{\prime}-1\right)\right) b
$$

holds, then it is a b.i.b. design and $v^{\prime}=v$.

Proof. Consider the first $v^{\prime}$ of the $v$ varieties. Let the $i$ th block have $x_{i}$ of these $v^{\prime}$ varieties, $i=1,2, \cdots, b$. As before

$$
\sum x_{i}=r v^{\prime}, \quad \sum x_{i}^{2}=r v^{\prime}+\lambda\left(v^{\prime}-1\right) v^{\prime} .
$$

So $r^{2} v^{\prime 2} \leqq\left(r+\lambda\left(v^{\prime}-1\right)\right) v^{\prime} b$. By (2) we infer that $r^{2} v^{\prime}=\left(r+\lambda\left(v^{\prime}-1\right)\right) b$. This implies that all the $x_{i}$ 's are equal; let $x_{i}=k, i=1,2, \cdots, b$.

If possible suppose $v^{\prime}<v$. Consider the $\left(v^{\prime}+1\right)$ th variety. As it appears with each of the first $v^{\prime}$ varieties in $\lambda$ of the blocks one obtains easily $r k=\lambda v^{\prime}$. Also $r^{2} v^{\prime}=r b k=\left(r+\lambda\left(v^{\prime}-1\right)\right) b$, that is, $r k=r$ $+\lambda\left(v^{\prime}-1\right)$. So we infer that $r-\lambda=0$. But this is impossible as $r=\lambda$ means that all the blocks are identical. Therefore $v^{\prime}=v$ and each block contains $k$ of the varieties. Thus the design is a b.i.b. design.

It can be shown by examples that the above theorem becomes false if we do not demand the appearance of all the varieties a constant number of times in the design.

\section{REFERENCES}

1. K. N. Majumdar, On some theorems in combinatorics relating to incomplete block designs, Ann. Math. Statist. 24 (1953), 377-389. 664.

2. - On combinatorial arrangements, Proc. Amer. Math. Soc. 5 (1954), 662-

3. H. J. Ryser, $A$ note on a combinatorial problem, Proc. Amer. Math. Soc. 1 (1950), 422-424.

DeLHI UNIVERSITY, INDIA 